\title{
THE ETHICAL ASPECT OF SCIENTIFIC INTEREST IN SELECTED PHYSICAL THEORIES
}

\begin{abstract}
Mainstream physicists do not form a unified group of scientists sharing the same opinions. It is generally known that their opinions on the dispute regarding the very foundations of quantum mechanics between Einstein and Bohr still differ. Many renowned physicists defend Einstein's standpoint. However, the support of Bohr's assertions regarding quantum mechanics by various grant agencies and foundations is also very significant. The present paper compares the two physical theories. In their case, there is basically no correlation with empirical indicators. The paper points out the robustness of the theory of strings and the theory of physical vacuum. Physicists elaborated both of these theories on a theoretical basis and they both have strong mathematical bases. Moreover, the theory of physical vacuum can be described as remarkably mathematically elegant. Despite the fact that neither of the theories has yielded relevant empirical results they continue to benefit from the lavish support they get. Witten's string theory is widely supported by mainstream institutions which provide funding to research. As Peter Woit points out it is due to the fact that the string theory is the only game in town. It was born in western culture and the richest institutions found many excellent mathematical physicists who studied the same field. The theory of physical vacuum is equally far-reaching. Its highly ambitious plan-geometrisation of the field using Einstein's method aims at developing a theory of field which would unify all hitherto physical interactions. Simultaneously, the theory introduces a new type of physical field, the so called primary torsion field, and extends the principle of the relativity theory. The theory, however, presents several erroneous results. Moreover, there are no empirical correlations and it has failed to achieve the success of theoretical predictions of the string theory. On the other hand, it receives almost no financial support from mainstream agencies and the number of scientists dealing with the theory is considerably lower. If this theory received as much financial support and scientific attention as the string theory, it would probably achieve the same empirical results. Financial resources and support from relevant institutions thus play a significant role in scientific research.
\end{abstract}

Keywords: robustness of the theory, support of research, theoretical physics, string theory, physical vacuum theory

\section{Introduction}

The issue of validity of scientific theories stands at the centre of attention of epistemology, philosophy of science, ethics of science and sociology of science. Nevertheless, new findings and new methodological approaches, stemming from these new scientific disciplines, are sometimes "difficult to promote because humans prefer the position of 'detached observers' (or, supposedly, 'objective scientists') to that of 'engaged participants' who, by their own attitudes and actions, influence the very process of knowing and thus the results of their scrutiny" [1, p. 25]. The neglect on the side of scientists of sociology of science and scientific ethics has resulted in inadequate methodologies, faulty data analyses, non - objective discriminatory practices, and even cases of fraud. This ethical conundrum, involving a preferential treatment of certain theories over others by scientific institutions as well as media would lead (if unchecked), as Soren Kierkegaard (1813 - 1855) prophesized over 150 years ago, to a societal decline [2 - 6]. Currently, natural sciences and predominantly physics give preference to particular groups of scientists who share common standpoints. Contemporary mainstream physics is, however, differentiated. It is no secret that opinions of physicists on the dispute between Bohr and Einstein regarding the foundations of quantum mechanics differ significantly. In this case, there is no winner. Both Bohr and Einstein have their adherents. However, there are certain lobbying interest groups which favour physicists who in this dispute defend standpoints close to Bohr's [7].

The present paper provides a critical analysis of two theories present in current physics and attempts to evaluate them from the viewpoint of general scientific criteria. The two theories are the

\footnotetext{
* ${ }^{1}$ Marian Ambrozy, ${ }^{2}$ Michal Valco, ${ }^{3}$ Suresh Bhattarai

${ }^{1}$ College of International Business ISM Slovakia in Presov, Duchnovicovo namestie 1, 080 01, Presov, Slovakia

${ }^{2}$ Faculty of Arts, Constantine the Philosopher University of Nitra in Nitra, Hodzova 1, 94974 Nitra, Slovakia

${ }^{3}$ Nepal Astronomical Society, House No. 1877, Bijay Chowk, Prayag Marg, Caulasa Kathmandu, 44600, Nepal

E-mail: mvalco@ukf.sk
} 
theory of strings and the theory of physical vacuum. The paper also analyses the position of the two theories in contemporary science in correlation with relevant features of serious scientific theories.

The reasons behind the search for a more unified and fundamental theory were the low ability of the general relativity theory to make predictions in micro space and the incompatibility of quantum theory with general relativity theory. "They were both successfully verified experimentally although each of them separately. They both deliver relatively precise adequate results and explain many new processes and phenomena. Nevertheless, both of them are incomplete and limited with respect to universe as a dynamic system" [8, p. 507]. Neither the standard model nor quantum mechanics can become a comprehensive physical description of the world.

\section{The results of string theory}

The string theory has been postulated this way since the 1970s. The new theory has an ambitious goal to unify all three independently existing interactions - electroweak, strong and gravitational. The theory of superstrings was originally formulated in twenty - six - dimensional space and it introduced physical particles tachyons. It was a very interesting phenomenon. Tachyons exceed the speed of light which is in contradiction to special relativity theory. In the mid - 1980s scientists worked with five different string theories. Then Edward Witten formulated the so - called M - theory. The aim of his ambitious attempt was to unify the existing string theories - I, II A, II B, heterotic O type and heterotic $\mathrm{E}$ type. The $\mathrm{M}$ - theory formulated by Witten is considered the mother theory [9].

One theory united the five then known string theories into one. String theory II A includes closed superstrings and I SO (32) includes open superstrings. The two theories are connected through string dualities. They are the S - duality and $\mathrm{T}$ - duality. One of the unified theories assumes that there are gravitinos which are supersymmetric particles to gravitons. Reduction of the ten - dimensional string theory to a six - dimensional variety is the way to achieve four - dimensional space - time. It is a six dimensional Calabi - Yau variety. Physicists defending the string theory believe that the same process can be observed in nature too.

String theoreticians think that the robustness and explanatory power of the string theory rests in the fact that it can explain the variety of particles of the standard model on the basis of transformations of vibrating points [10]. Mathematical unification of the string theory is truly remarkable. It exceeds the standard model and unifies the three interactions in accordance with Comte's or Laudan's dream (compare [11]). The string theory helps explain the invariant for the black hole entropy. "Deriving the thermos - dynamic features of black holes from the string theory persuaded many indecisive physicists that the theory of strings is on the right path to understanding the quantum aspects of gravity" [12, p. 285]. P - gateways formulated by string theoreticians helped construct the black hole model while deriving of a formula for calculating the black hole entropy is compatible with mathematical calculations of the string theory. This is an undisputable success of the explanatory aspect of the string theory.

Strings are very interesting and from the explanatory viewpoint also highly successful theories. However, the same cannot be said about the empirical correlation of theory and results. There are basically no empirical confirmations of the string theory. In comparison with the standard model the string theory loses because in the case of the standard model there are clear correlations between experiment and prognosis. Projecting of perturbation theories was relatively successful; nevertheless, they often seem divergent. Perturbation theories are not controversial; however, their divergence prevents their ability to predict.

The question whether it is possible to empirically verify or falsify the string theory represents a serious problem. String theoreticians often argue that the length of their theoretical objects is almost identical with the Planck length and therefore it is not possible to observe these objects with currently available technology. It is a strong argument. On the other hand, however, it makes the entire theory empirically unverifiable. Based on the aforementioned Rudolf Carnap who was a radical logical empiricist declared the theory scientifically meaningless. The present paper does not share his radicalism. Principally, it is not an unverifiable theory. Arguments speaking of the beauty of unification of the general relativity theory and standard model can be considered shallow. The elegance of the solution according to which one - dimensional strings vibrate with different energies, which explains the variety of particles in the standard model, is not a real argument. One cannot speak of the existence of string equations because there are no such equations. Mathematical background of the theory is questionable. Transfer of the eleven - dimensional space of the $\mathrm{M}$ - theory to four - dimensional space - time represents a problem.

The real problem is relativisation of space - time which has gone beyond the relativisation of the general relativity theory. In general relativity theory space - time is of the Pseudo - Riemannian variety. Its relativisation has certain boundaries, it cannot be perforated or pierced and it cannot have bizarre features. The string theory enables hitherto unimaginable operations with space - time. "The string theory knows space - time rupture, perforation, ripping and sewing up etc., the so - called flops" [13, p. 702]. It is interesting that in the string theory space - time has a substantial not relational character, which brings the theory closer to Newton than to Einstein. Changes of space - time happen in dynamic reality. The strings can be even reeled up.

What is the M - theory? It is very difficult to obtain a consistent answer to this question. The answer should 
unify all five superstring theories. M - theory tries to explain spontaneous disruption of symmetry as well as eliminate faulty development from partial theories. Its correct formulation is thus problematic. The string theory has provided some predictions regarding hypothetical particles and new types of symmetries. However, they are just theoretical constructs since none of the abovementioned has been observed. Within the boundaries of empirical correlation, the explanatory power of the theory is very low. Fault theory is very approximate. As stated above the only thing the string theory has really explained is the construction of black holes by means of $\mathrm{p}$ - gateways.

If the string theory depicts its objects one dimensionally, it comes close to point images of the standard model. No new particles predicted by the string theory have been detected yet. The relatively promising explanatory successes of the theory have not been supported by any observation. It can be said that scientists have not confirmed any direct connection between empirical data and predictions of the string theory. From the viewpoint of physics, it is important to add that verification of the theory's predictions is actually possible. However, nothing has been confirmed yet. Therefore, we speak of unconfirmed and not meaningless theory. Although the string theory has been known for more than thirty years, physicists have not presented a single significant empirical correlation of this theory. Against the string theory is also the fact that there can be so many of them [14], and their number can grow to a really large figure $[15$, p. 3].

So how can a theory with such poor empirical successes keep its position in mainstream physics? The famous physicist Peter Woit explains it by his statement that it is the "only game in town" [16]. It is absolutely clear that it is sociology rather than physical arguments which can explain these reasons. Perhaps it could be said that the predictive power of the $\mathrm{M}$ - theory is weaker than we expected [15, p. 56]. In our opinion this theory is only influential due to sociological factors and has achieved only partial success in the field of theoretical physics. However, one cannot speak of any success in the field of experimental physics. The string theory owes its success to the interest of many renowned physicists followed by the support of relevant institutions and lobbying groups. Currently, the world of science is more and more aware of the theory's weaknesses, its inability to predict observed phenomena and verify predictions.

The future needs to bring further relevant experiments which can or cannot refute the theory whereas the latter would mean its temporary verification. However, considering the hitherto empirical indicators and enormous possibilities regarding string theory variety this scenario is quite improbable.

\section{The results of the theory of physical vacuum}

Another well - developed theory which aims at becoming a physical theory of everything is the theory of physical vacuum.
The fathers of this Russian physical theory are the physicists A. E. Akimov and G. I. Shipov. The - well known physicist academician Pomeranchuk said that the future belongs to the physics of the vacuum. G. I. Shipov developed physics of the vacuum to be in accordance with the theory of physical vacuum. He considers physical vacuum the basis of physics claiming that "it was proven on the levels of vacuum that compliance of central basic levels of physics is done in accordance with them" [17, p. 18]. For Shipov the excited state of physical vacuum is a special state of emptiness. He tries to formulate a unified theory of the field by means of geometrisation of equations of the electromagnetic field. The basis of this geometrisation is application of geometry of absolute parallelism which is connected with rotating systems and Weizenbeck geometry.

Shipov introduced a new type of field - primary torsion field. "In special scientific meaning the category of field shall mean its representation in the conceptual system of physical sciences, it is the category which expresses mutual influence of material physical objects" [18, p. 757]. It is Weizenbeck geometry which describes both a space curved in a non - Euclidian manner and the rotation of the space. Space in which the physical vacuum is described by the abovementioned geometry has ten dimensions. Initial active vortexes cause the space to bend whereas material sources are reduced. What remains active is the excited vacuum. The core of the scientific programme of G. I. Shipov and his colleagues is the geometrisation of space. Shipov explains the emergence of physical reality as changes of space curves and space rotation.

The theory of physical vacuum structures physical reality into several levels. The first level is the level of absolute nothingness. Nothing concrete can be said about this level, and yet it has enormous creative possibilities. This level is mathematically described by a system of equations where the Riemann curve turns to zero [17, p. 82]. It resembles Hegel's Pure Nothing from his work Science of Logic. The second level is the primary torsion field which originates from the first level. This field resembles Parmenidean full existence which a priori excludes the existence of non - being. It is subtle matter reality. Primary torsion field is present everywhere because its energy and impulse equal zero. Other levels are connected with plasma, gas, liquid and solid states of matter. The state of matter depends on the tempo of quantum vibration.

The theory of physical vacuum also includes tachyons, i.e. particles which exceed the speed of light. The theory also mentions the existence of particles with reverse time run, mass and anti - mass which emerge from polarised torsion field. The theory also presents the category of imaginary rest mass. It is also necessary to mention Terlecky's quadrigas and Akimov's fitons. These micro particles predicted by the theory of physical vacuum should resemble de Broglie's corpuscular wave dualism. In accordance with the conclusions of the EPR paradox our theory 
claims that speed of information transmission can be immediate and can thus exceed the speed of light.

The theory's plan is to become the physical theory of everything by elaborating a theoretical basis for unification of all interactions. The field of inertia can include all forms of matter. Naturally this would mean the unification of the quantum theory with the general relativity theory. Physical vacuum theoreticians often try to apply the principle of correspondence in the field of quantum theory whereas they deem basic physical equations to be special cases of equations of the torsion field. The adherents of the theory believe that the deductive theory of the field means further development of the quantum theory in accordance with Einstein's ideas. Comprehensive wave function and Schrödinger's equation comply. The newly introduced "quantum constant (an analogy to the Planck constant $h$ ) seems to be an arbitrary constant the value of which is (probably) determined by studying the geometrical parameters of the observed system" [17, p. 173]. In Shipov's opinion the theory has a deterministic character.

A critical review of the theory of physical vacuum reveals several problems. Adherents of the theory claim that it is supported by experiments which confirm it $[19,20]$. Critics of the theory state that explanation of the experiments is incorrect. V. A. Rubakov says that the results can be explained by Newtonian mechanics and Maxwell's electrodynamics. Shipov, however, "offers an "experimental proof" of not preserving the impulse in mechanics and then draws a picture of motion on a new transport with "torsion motion"” [21, p. 351]. He similarly criticises the origination of Terlecky's quadrigas. They originate as a result of uniting particles with positive and negative weight which in Rubakov's opinion is in contradiction to all hitherto experiments. Shipov sees new potential of electromagnetic field in equations of geometrised electrodynamics. Rubakov criticises Shipov's three basic equations. Shipov believes they are the basis of physics whereas Rubakov describes them as mathematically elegant theories with no relation to physical reality. On the other hand, it is necessary to say that the existence of torsion fields was thought possible before the occurrence of the theory of physical vacuum. If they do exist, most physicists believe that their manifestations are very weak. Now scientists face a question whether it is possible to experimentally verify the core of the theory which is the existence of primary torsion field. Facts show that "with current level of experiments it is not possible to register torsion fields" [22, p. 35]. Akimov's assertion that it is possible to generate energy from vacuum has not been confirmed either. The theory of physical vacuum presents several erroneous conclusions. Declared correlations with experiments can be explained with the help of established physical theories. It is necessary to emphasise the fact that currently there are no means which could confirm or refute the existence of primary torsion field. Applying the philosophy of science of W. V. O. Quine [23], one must say that at present it is not possible to falsify statements attacking the core of the theory of physical vacuum.
The string theory eliminates discontinuity of points because the size of a string is limited. From the viewpoint of ontology of science, it is a step forward that first bases actually receive more concrete attributes than idealised monads resembling point objects studied by mathematics of the standard model. While the string theory is, in a way, based on Newtonian' principles of paradigm (substantial nature of space - time, inertial frame of reference), Shipov, in the theory of physical vacuum, refers also to Descartes [24] when he says that all movement is rotation. Twisting of space in accordance with Riemann - Cartan geometry is accepted by string theoreticians, however, these effects cannot be studied by currently available technologies. Nevertheless, torsion effects predicted by geometry of absolute parallelism have no recorded experimental results either. Conclusions of experiments quoted by Akimov and Shipov can be deduced from other physical principles. The other possibility is that invariant real results of experiments do not exist at all.

The mathematical background of the string theory is very strong and can explain theoretically better - known facts. The five string theories can be considered consistent. They do not contradict known physical facts and they probably do not contain mistakes that the critics identified in the theory of physical vacuum. On the other hand, however, there are far more physicists studying the string theory than those studying the theory of physical vacuum. What is missing, however, are the real correlations with physical reality supported by experiments. Again, one cannot speak of anything else than of a physical theory ungrounded by facts.

As Mrozek said, mathematical elegance cannot be the only criterion determining the success of physical theory [25]. First of all, it is necessary to find out whether experiments comply with predictions and mathematically described theory. Preserving the causality principle is also necessary. It is assumed that advancing technologies will enable falsification or verification of the compared theories and that it will be possible to reliably confirm or falsify the predicted objects in the future. These objects are not considered unverifiable. However, it is believed that our civilisation does not have the necessary means to do so.

\section{Conclusion}

Comparison of the success rates of the physical vacuum theory and the string theory slightly favours the string theory. The cores of both theories are consistent and have solid mathematical foundations; however, currently it is not possible to falsify them experimentally. With respect to certain theoretical results and higher level of consistency with established physical theories the string theory emerges as slightly more successful. The relationship between the two theories is apparently contradictory and so too is the tertium non datur situation in the case of their future falsification or verification. It is quite possible that eventually 
both theories will be falsified and a new ontological basis having the attributes of fundamental ontological entities will appear on the scene. The sociology of science reacts with a counterargument pointing at totally uneven support for the two theories on the part of mainstream agencies, institutions, scientific committees of prestigious journals, etc. There is a striking difference in the number of scientists dealing with these two physical theories. We dare to say that the string theory, as the better known "only game in town", owes its success to sociological factors, support of scientific institutions and renowned scientific publishing houses as well as to enormous financial support. We believe that with equal share of attention, amount of scientific work and financial resources and particularly with involvement of an equivalent number of renowned physicists the theory of physical vacuum could achieve more or less the same scientific results. The fact that the results achieved are still quite poor and lack connection to experimental measuring is the problem of sociology of science and scientific ethics. Any support of scientific research that has no real experimental results is not only bizarre but potentially detrimental for further research and collaboration among scientists. The selectiveness of this support determined by other than strictly epistemological reasons is a strange aspect of scientific life which often steers declared freedom of scientific research towards Hegelian understanding of freedom as recognition of necessity. In this case it can also be noted, that change can be understood as a new impulse that will provide an opportunity for a genuine creativity, provide more space for application of ideas, and even those not following only mainstream wave [26]. Enabling full freedom of scientific research supported by the interest and funding of responsible institutions could thus acquire a more realistic direction.

This paper was written as part of the tasks of the "Selected philosophical and ethical consequences of theoretical physics", supported by institutional grants IG-KSV-01/2016/2.1.5 and VTS -> 3509412 Z-15-102/0013: Kierkegaard and Classical Greek Thought.

\section{References}

[1] VALCO, M., VALCOVA, K.: The Epistemological Challenge of Kierkegaard's Truth is Subjectivity Principle. Communications Scientific Letters of the University of Zilina, 16(3), 25-28, 2014.

[2] This topic has become a prominent subject of analyses among contemporary Kierkegaardian scholars. See: KRALIK, R., TINLEY, S. J.: Kierkegaard's Ethics as an Answer to Human Alienation in Technocratic Society. Communications - Scientific Letters of the University of Zilina, 19(1), 25-29, 2017; ZALEC, B. Preferential and Non - Preferential Love in Light of Kierkegaard's Thought. European Journal of Science and Theology, 13(2), 179-189, 2017.

[3] PAVLIKOVA, M.: Reading Auden as Resource for Existential Reflection in a Society with Technocratic and Hedonistic Tendencies. Communications - Scientific Letters of the University of Zilina, 19(1), 25-29, 2017; KONDRLA, P. \& REPAR, P.: Ontological Consequences of the Ethics of Technology. Communications - Scientific Letters of the University of Zilina, 19(1), 19-24, 2017.

[4] VAlCOVA, K., PAVLIKOVA, M., ROUBALOVA, M.: Religious Existentialism as a Countermeasure to Moralistic Therapeutic Deism. Communications - Scientific Letters of the University of Zilina, 18(3), 98-104, 2016; KRALIK, R.: Kierkegaard's Interpretation of Faith. XLinguae, 10(3), 37-44, 2017.

[5] VALCO, M., KRALIK, R., BARRETT, L.: Moral Implications of Augustine's Philosophical and Spiritual Journey in his Confessiones. Communications - Scientific Letters of the University of Zilina, 17(2), 103-108, 2015; KONDRLA, P. \& REPAR, P.: Postmodern Aspects of New Religious Movements. European Journal of Science and Theology, 13(3), 67-74, 2017.

[6] PAVLIKOVA, M.: Aspects of Mortality in Literary Work of Don DeLillo. XLinguae, 10(1), 62-69, 2017; also see receptions: KRALIK, R.: Reception of Søren Kierkegaard in Czech language (in Czech). Filosoficky casopis, 61(3), 443-451, 2013; KRALIK, R., PAVLIKOVA, M.: The Reception of Kierkegaard's Work in Slovakia. Filozofia, 68(1), 82-86, 2013.

[7] PROCHAZKA, J., LOKAJICEK, M. V., KUNDRAT, V.: Dependence of Elastic Hadron Collisions on Impact Parameter. The European Physical Journal Plus, 131(5), 1-19, 2016.

[8] DUBNICKA, J.: Space, Time and Quantum Gravity. Organon F, 13(4), 506-523, 2006.

[9] WITTEN, E.: Superconducting Strings. Nuclear Physics Section B, 249(4), 557-592, 1985.

[10] GREENE, B.: The Elegant Universe. Vintage Series, Random House, UK, 2000.

[11] KARABA, M.: Laudan's Reticulational Model of Scientific Progress (in Slovak). Filosoficky casopis, 60(4), 519-535, 2012.

[12] MOTL, L.: Dream about Quantum Gravitation or a Brief History of M - Theory. Pokroky matematiky, fyziky a astronomie, 48(4), 277-287, 2003.

[13] DUBNICKA, J.: The String Theory and Physical Worldview. Filozofia, 63(8), 695-703, 2008.

[14] KOSTELECKY, V. A., SAMUEL, S.: Phenomenological Gravitational Constraints on Strings and Higher - Dimensional Theories. Physical Review Letters, 63(3), 224-227, 1989. 


\section{KOMNIKCCle}

[15] DOUGLAS, M. R.: The Statistics of String/M Theory Vacua. Journal of High Energy Physics, 7(5), 1077-1137, 2003.

[16] WOIT, P.: Not Even Wrong: The Failure of String Theory and the Continuing Challenge to Unify the Laws Of Physics. Random House, UK, 2011.

[17] SHIPOV, G. I.: Theory of Physical Vacuum (in Russian). HT - Centr, Moskva, 1993.

[18] DUBNICKA, J.: The Theory of Quantum Gravitation and the Theory of Relativity. Filozofia, 66(4), 325-335, 2011.

[19] AKIMOV, A. E., SHIPOV, G. I.: Torsions Fields and their Experimental Manifestations. Journal of New Energy, 2(2), 1997, 67-84.

[20] AKIMOV, A. E., TARASENKO, V. Y., TOLMACHEV, S. Y.: Torsion Communication. A New Physical Base Information Transmission Systems. Elektrosvyaz, 5, 24-30, 2001.

[21] RUBAKOV, V. A.: About the Book by G. I. Shipov 'Theory of Physical Vacuum'. Theory, Experiments, and Technology (in Russian). Uspechi fyziceskich nayk, 170(3), 35-352, 2000.

[22] EYDELMAN, E. D.: Science and Pseudoscience under the Microscope of Science (in Russian). Zdravij smysl, 11(2), 35.2006.

[23] QUINE, W. V.: On Empirically Equivalent Systems of the World. Erkenntnis, 9(3), 313-328, 1975.

[24] DESCARTES, R.: Principles of Philosophy. BookRix, 2017.

[25] MROZEK, J.: Is There Retreat from Naturalism in Physics? Proceedings of 2nd International Multidisciplinary Scientific Conference on Social Sciences and Arts (SGEM 2015), Bulgaria, 2015.

[26] POLACKO, J., STOROSKA, M.: Change as Opportunity - One of the Elements of the Progress in Management. Proceedings of the International Scientific Conference Renewable Energy Sources - Potential, Economy, Character and Technology (RESpect 2011), Technical University of Kosice, Slovakia, 2011. 